\title{
至 SpringerLink
}

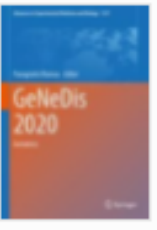

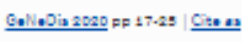

Greek Nurses' Perception of Hospital Ethical Climate: A Cross-Sectional Study

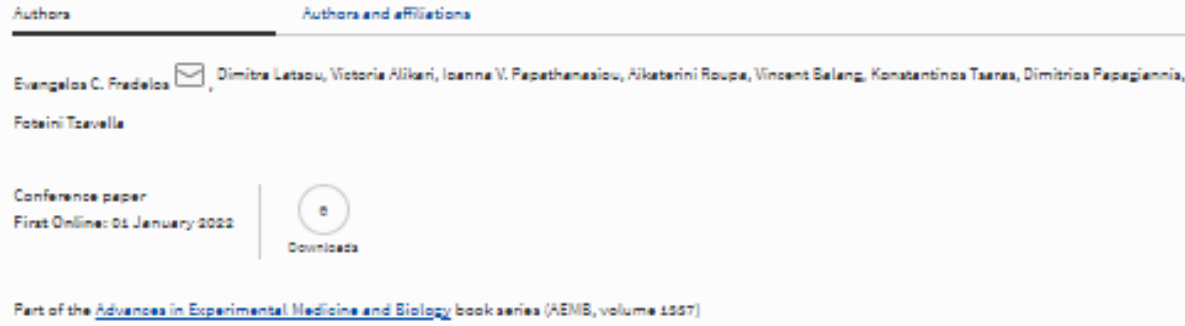

Abstract

This study aimed to examine Greek nurses' perceptions about hospital ethical climate and to investigate the possible difference of those perceptions regarding their demographic and work-related characteristics. The cross-sectional study design was employed in this study in which 286 nurses and nurse assistants participated. Data were collected by a sheet containing demographic and work-related characteristics and the Greek version of the Oslons' Hospital Ethical Climate Scale. IBM Statistical Package for Social Sciences 25 was used in data analysis. Frequencies, means, percentages, and standard deviations summarized the data. For the statistical differences, parametric tests were performed. Independent Samples $t$ and pearson correlation analysis were used to determine the relationship between the ethical clinate of the hospital and the nurses' characteristics. The $p$-values 0.05 were considered statistically significant The mean age of the nurses was 44 years (SD: 8.5 years; range $24-66$ years). The majority of them were women ( $77.3 \%)$. A percent of $57.7 \%$ of the sample was married. Most positive perceptions were conoerning managers ( 4.01 ) following by peers (3.82), patients (3.69), hospitals (3.29) while the least positive percoptions of the ethical climate were concerning the physicians (3.16). The factors associated with hospital ethical perception were working experience and responsible position. The highest score of ethical climate reported to managers subscale, while the minimum score was related to physicians. In general, Greek nurses reported positive perceptions regarding hospital ethical dimate. The positive ethical climate is associated with a better working environment, fewer nurses' experience of moral distress, fener chances for nursing turnover, high quality of nursing care, and fever errors in nursing practice

Keywords 


\title{
Title: Greek nurses' perception of hospital ethical climate. A cross-sectional study \\ Subtitle: Ethical climate in Greek Hospitals \\ Authors
}

1. Evangelos C. Fradelos, Internal Medicine Department, Athens General Hospital of Chest Diseases "Sotiria", Greece

2. Dimitra Latsou, Department of Social and Educational Policy, University of Peloponnese, Greece

3. Victoria Alikari, Lecturer, Department of Nursing, University of West Attica, Greece

4. Papthanasiou V. Ioanna, Nursing Department, University of Thessaly, Greece

5. Aikaterini Roupa, Children ICU, University Hospital of Herakleion, Greece

6. Vincent Balang, Department of Nursing, University Malaysia Sarawak, Malaysia.

7. Konstantinos Tsaras, General Department, University of Thessaly, Greece

8. Dimitrios Papagiannis, Nursing Department, University of Thessaly, Greece

9. Foteini Tzavella, Nursing Department, University of Peloponnese, Greece

Correspondent author: Evangelos C. Fradelos, E-mail: efradelos@uth.gr

\begin{abstract}
The present study aimed to examine Greek nurses' perceptions about hospital ethical climate and to investigate the possible difference of those perceptions regarding their demographic and work-related characteristics. The cross-sectional study design was employed in this study in which 286 nurses and nurse assistants participated. Data were collected by a sheet containing demographic and work-related characteristics and the Greek version of Oslons' Hospital Ethical Climate Scale. IBM Statistical Package for Social Sciences 25 was used in data analysis. Frequencies, means, percentages, and standard deviations summarized the data. For the statistical differences, parametric tests were performed. Independent Samples $t$ and Pearson correlation analysis were used to determine the relationship between the ethical climate of the hospital and the nurses' characteristics. The p-values 0.05 were considered statistically significant. The mean age of the nurses was 44 years (SD: 8.5 years; range 24-66 years). The majority of them were women $(77.3 \%)$. A percent of $57.7 \%$ of the sample was married. Most positive perceptions were concerning managers (4.01) following by peers (3.82), patients (3.69), hospital (3.29) while the least positive perceptions of the ethical climate were concerning the physicians (3.16). The factors associated with hospital ethical perception were: working experience and responsible position. The highest score of ethical climate reported to Managers subscale, while the minimum score was related to Physicians. In general, Greek nurses reported positive perceptions regarding hospital ethical climate. The positive ethical climate is associated with a better working environment, fewer nurses' experience of moral distress, fewer chances for nursing turnover, high quality of nursing care, and fewer errors in nursing practice.
\end{abstract}

Key words: Nurses, Hospital ethical climate, Hospital environment

\section{Introduction}

Organizational climate is the "psychological atmosphere" of the organization's environment, as it is perceived and experienced by its members, and it appears to exert influence on their behavior. It is the feeling of comfort or disgust with the particular work environment and it motivates the conditions of high or low job satisfaction and performance. According to studies [1,2], the organizational climate is the way members of the organization perceive and characterize the organizational environment. It is 